\title{
LOCAL STATISTICAL FEATURES OF DUAL TREE COMPLEX WAVELET TRANSFORM BASED FACE RECOGNITION WITH SINGLE SAMPLE
}

\author{
Jaya Priya .K' , Dr. Rajesh .R.S ${ }^{2}$ \\ ${ }^{1}$ Research Scholar, Mother Teresa Women's University, INDIA \\ ${ }^{2}$ Department of Computer Science and Engineering, Manonmaniam Sundaranar University, INDIA \\ E-mail : 'kjp.jayapriya@yahoo.com
}

\section{Abstract}

In this paper, we propose novel face recognition method based on local appearance feature extraction using dual-tree complex wavelet transform (DT-CWT). It provides a local multiscale description of images with good directional selectivity and invariance to shifts and in-plane rotations. In the dual-tree implementation, two parallel discrete wavelet transform (DWT) with different lowpass and highpass filters in different scales are used. The linear combination of subbands generated by two parallel DWT is used to generate 6 different directional subbands with complex coefficients. It is insensitive to illumination variations and facial expression changes. 2-D dual-tree complex wavelet transform is less redundant and computationally efficient. The local DT-CWT coefficients are used to extract the facial features which improve the face recognition with small sample size in less computation. The local features based methods have been successfully applied to face recognition and achieved state-of-the-art performance. Normally most of the local appearance based methods the facial features are extracted from several local regions and concatenated into an enhanced feature vector as a face descriptor. In this approach we divide the face into several $(\mathrm{m} \times \mathrm{m})$ non-overlapped parallelogram blocks instead of square or rectangle blocks. The local mean and standard deviation of complex wavelet coefficients are used to describe the face image. Experiments, on two well-known databases, namely, Yale and ORL databases, shows the Local DT-CWT approach performs well on illumination, expression and perspective variant faces with single sample compared to PCA and global DT-CWT. Furthermore, in addition to the consistent and promising classification performances, our proposed Local DT-CWT based method has a really low computational complexity.

Keywords: Gabor Wavelet Transform, Dual Tree Discrete Wavelet Transform, Dual Tree Complex Wavelet Transform, PCA, Parallelogram Regions.

\section{INTRODUCTION}

In recent years face recognition received more attention in the field of biometric authentication. This is due to increased concerns in security. However, the general problem of face recognition remains to be solved, since most of the systems to date can only successfully recognize faces when images are obtained under prescribed conditions. Their performance will degrade abruptly when face images are captured under varying pose, lighting, with accessories and expression. Another one of the most challenging problems for face recognition is the so-called Single Sample Problem (SSP) problem, i.e., a single face for a subject is used for training. Large training samples can not be guaranteed in practice such as identity card verification, passport verification, etc. Some face recognition algorithms have been proposed to solve the face recognition problem with only a single training image with various mode of process [1]. A face recognition system should, to a large extent, take into account all the above-mentioned natural constraints and cope with them in an effective manner. In order to achieve this, one must have efficient and effective representations forfaces.

Many techniques have been proposed in the literature for representing face images. Some of these include principal components analysis [2], discrete wavelet transform [3, 4], and discrete cosine transform [5]. Gabor wavelet-based representation provides an excellent solution when one considers all the above desirable properties. For this reason, Gabor wavelets have been extensively implemented in many face recognition approaches [6-9].

Even though Gabor wavelet-based face image representation is optimal in many respects, it has got two important drawbacks that shadow its success. First, it is computationally very complex. A full representation encompassing many directions (e.g., 8 directions), and many scales (e.g., 5 scales) requires the convolution of the face image with 40 Gabor wavelet kernels. Second, memory requirements for storing Gabor features are very high. The size of the Gabor feature vector for an input image of size $128 \times 128$ pixels is $128 \times 128 \times 40=655360$ pixels when the representation uses 8 directions and 5 scales.

Recently wavelets under multiresolution framework have been shown to help combat the detrimental effects of both noise and non-uniform illumination [10] in face recognition and facial feature extraction. The dual tree implementation of complex wavelet transform provides a directional multiresolution decomposition of a given image 
much like the Gabor wavelets however unlike the Gabor wavelets it has relatively less computational expense. Thus, complex approximately analytic wavelets provide an excellent alternative to Gabor wavelets with the potential to overcome the above-mentioned shortcomings of the Gabor wavelets.

In this paper we propose a new approach to improve the robustness of DT-CWT for pose and expression invariant face recognition with small sample size. This approach is based on local magnitude of the DT-CWT coefficients. In order to capture multi-orientation information in face images better. Therefore, each face image is described by a subset of band filtered images containing DT-CWT coefficients which characterize the face textures. We divide the DT-CWT sub-bands into small sub-blocks, from which we extract compact and meaningful feature vectors using simple statistical measures.

The rest of the paper is organized as follows. Sections 2 briefly give an overview of Gabor wavelets and DT-CWT. Section 3 describes the proposed method, and Section 4 which compare the performances of Local DT-CWT on parallelogram image structures approach with DT-CWT on the pose, illumination and expression variant faces of Yale and ORL datasets. Finally, conclusions are drawn in section 5 .

\section{RELATED WORK}

\section{A. Gabor Wavelet Transform}

A Gabor wavelet filter is a Gaussian kernel function modulated by a sinusoidal plane wave:

$$
\begin{aligned}
& \Psi_{g}(x, y)=\frac{f^{2}}{\eta \gamma \pi} \exp \left(\beta^{2} y^{\prime^{2}}-\alpha^{2} x^{\prime^{2}}\right) \exp \left(2 \Pi j f x^{\prime}\right) \\
& x^{\prime}=x \cos \theta+y \sin \theta \\
& y^{\prime}=y \cos \theta-x \sin \theta
\end{aligned}
$$

where $f$ is the central frequency of the sinusoidal plane wave, $\theta$ is the anticlockwise rotation of the Gaussian and the envelope wave, $a$ is the sharpness of the Gaussian along the major axis parallel to the wave, and $\beta$ is the sharpness of the Gaussian minor axis perpendicular to the wave. $\gamma=f / a$ and $\eta=f / \beta$ are defined to keep the ratio between frequency and sharpness constant. The 2D Gabor wavelet as defined in (1) has Fourier transform:

$$
\begin{aligned}
& \psi_{g}(u, v)=\exp \left(\pi^{2}\left(\frac{\left(u^{\prime}-f^{\prime}\right)^{2}}{\alpha^{2}}+\frac{v^{\prime 2}}{\beta^{2}}\right)\right) \\
& u^{\prime}=u \cos \theta+v \sin \theta, \\
& v^{\prime}=v \cos \theta-u \sin \theta .
\end{aligned}
$$

Figures $1(a)$ and $1(b)$ show, respectively, the real part and magnitude of the Gabor wavelets for 4 scales and 6 directions. Gabor wavelets possess many properties which make them attractive for many applications. Directional selectivity is one of the most important of these properties. The Gabor wavelets can be oriented to have excellent selectivity in any desired direction. They respond strongly to image features which are aligned in the same direction and their response to other feature directions is weak. Invariance properties to shifts and rotations also play an important role in their success. In order to accurately capture local features in face images, a space frequency analysis is desirable. Gabor functions provide the best trade off between spatial resolution and frequency resolution. The optimal frequency-space localization property allows Gabor wavelets to extract the maximum amount of information from local image regions. This optimal local representation of Gabor wavelets makes them insensitive and robust to facial expression changes in face recognition applications. The representation is also insensitive to illumination variations due to the fact that it lacks the DC component. Last but not least, there is a strong biological relevance of processing images by Gabor wavelets as they have similar shapes to the respective fields of simple cells in the primary visual cortex.

Despite many advantages of Gabor wavelet-based algorithms in face recognition, the high-computational complexity and high memory capacity requirement are important disadvantages. IWith a face image of size $128 \times$ 128 , the dimension of the extracted Gabor features would be 655360 , when 40 wavelets are used. This feature is formed by concatenating the result of convolving the face image with all the 40 wavelets. Such vector dimensions are extremely large and, in most cases, downsampling is employed before further dimensionality reduction techniques such as PCA is applied. The computational complexity is high even when fast Fourier transform (FFT) is employed. Because of the above-mentioned shortcomings, one usually looks for other transforms that can preserve most of the desired properties of Gabor wavelets and at the same time reduces the computational complexity and memory requirement. Complex wavelet transforms provide a satisfactory alternative to this problem. 


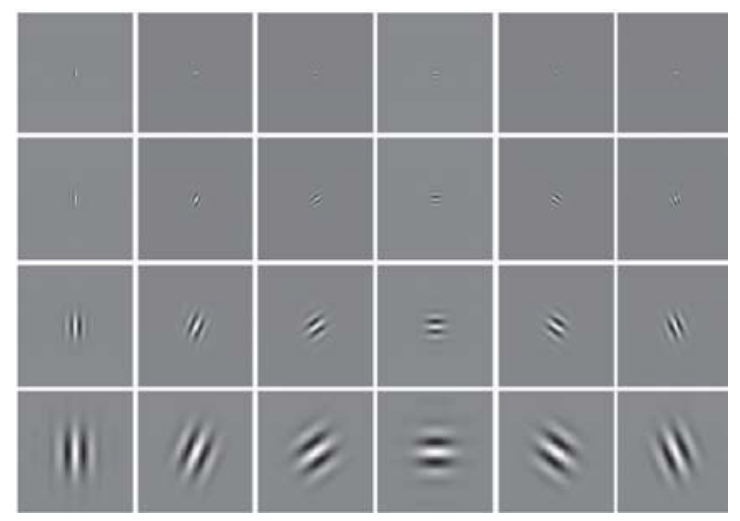

(a)

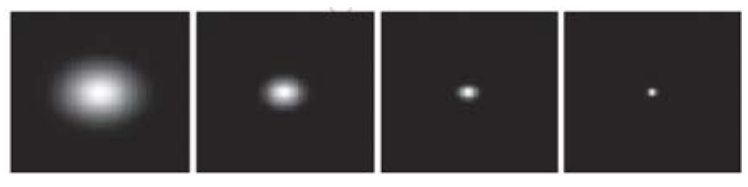

(b)

Fig. 1. Gabor wavelets. (a) The real part of the Gabor kernels at four scales and six orientations.

(b) The magnitude of the Gabor kernels at four different scales.0 0.1 0.2 0.3 0.4 0.5 0.6 0.7 0.8 0.91

\section{B. Dual-Tree Complex Wavelet Transform}

One of the most promising decompositions that remove the above drawbacks satisfactorily is the dual-tree complex wavelet transform (DT-CWT)[11-13]. Two classical wavelet trees (with real filters) are developed in parallel, with the wavelets forming (approximate) Hilbert pairs. One can then interpret the wavelets in the two trees of the DT-CWT as the real and imaginary parts of some complex wavelet $\Psi_{\mathrm{c}}(\mathrm{t})$. The requirement for the dual-tree setting for forming Hilbert transform pairs is the wellknown half sample delay condition. The resulting complex wavelet is then approximately analytic (i.e., approximately one sided in the frequency domain). The properties of the DT-CWT can be summarized as

a) approximate shift invariance;

b) good directional selectivity in 2 dimensions;

c) phase information;

d) perfect reconstruction using short linear-phase filters;

e) limited redundancy, independent of the number of scales, $2: 1$ for $1 \mathrm{D}$ ( $2 \mathrm{~m}: 1$ for $\mathrm{mD})$;

f) efficient order- $\mathrm{N}$ computation-only twice the simple DWT for 1D (2m times for $\mathrm{mD})$.

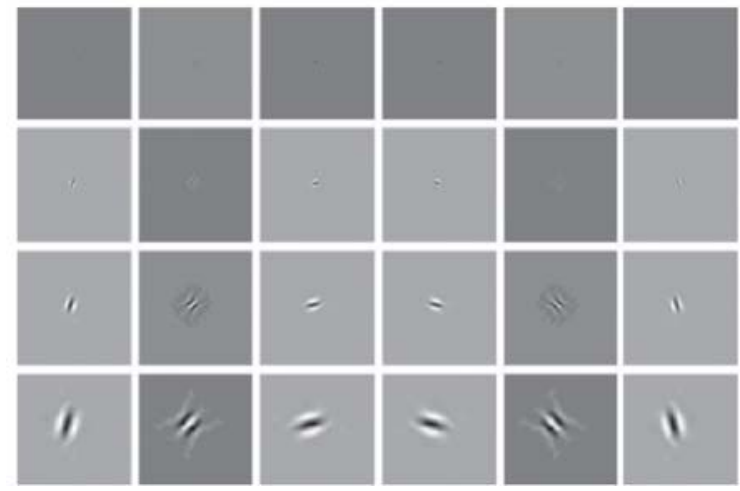

(a)
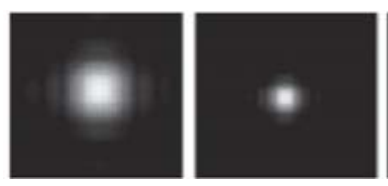

(b)

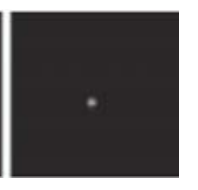

b)
Fig. 2. Impulse response of dual-tree complex wavelets at 4 levels

and 6 directions. (a) Real part. (b) Magnitude.

It has the ability to differentiate positive and negative frequencies and produces six subbands oriented in $\pm 15, \pm$ $45, \pm 75$. However, these directions are fixed unlike the Gabor case, where the wavelets can be oriented in any desired direction. Figure 2 shows the impulse responses of the dual-tree complex wavelets. It is evident that the transform is selective in 6 directions in all of the scales except the first. Figure 3 shows the magnitude and real part of a face image processed using the DT-CWT.

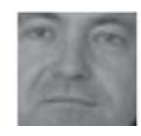

(a)
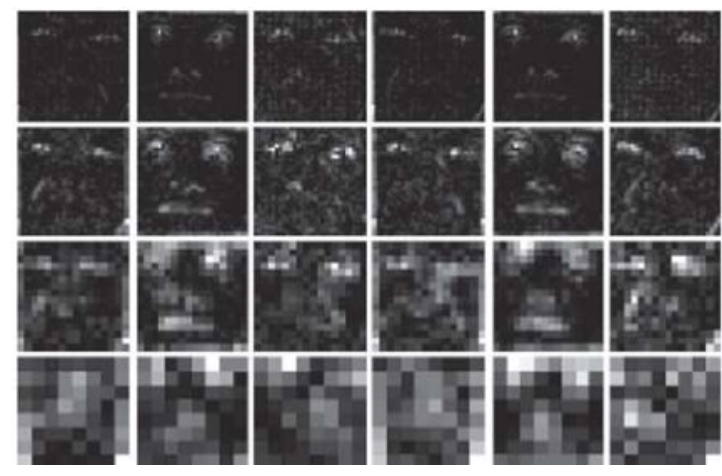

(b) 


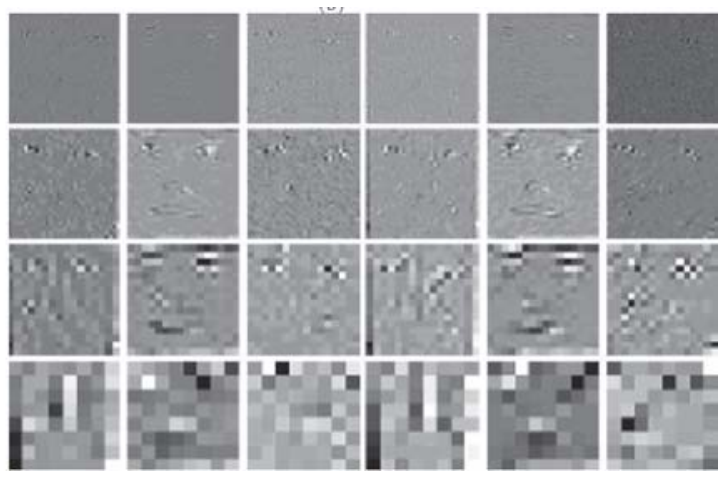

(c)

Fig. 3. (a) Sample image for transformation. (b) The magnitude of the transformation. (c) The real part of the transformation.

\section{LOCAL DUAL TREE COMPLEX WAVELET TRANSFORM BASED FACE REPRESENTATION}

First the directional multiscale decomposition of the gray level face image is performed up to level 4 . In order to extract the most discriminative features, first each subband is partitioned into a set of equally-sized blocks as well as parallelogram blocks in a non-overlapping way. The statistical measures such as mean and variance of the energy distribution of the magnitude of the complex coefficients for each sub-band at each decomposition level are used to represent the facial description.

Let $l_{i j}(x, y)$ be the image at the specific block $j$ of sub-band $\mathrm{i}$, the resulting feature vector $\mathrm{v}_{\mathrm{ij}}=\left\{\mu_{\mathrm{ij}}, \sigma_{\mathrm{ij}}^{2}, \mathrm{e}_{\mathrm{ij}}\right\}$, where $\mu_{\mathrm{ij}}=$ mean, $\sigma^{2}=$ variance.

$$
\begin{array}{r}
\mu_{i j}=\frac{1}{M \times N} \sum_{x=1}^{M} \sum_{y=1}^{N}\left|I_{i j}(x, y)\right| \\
\sigma_{i j}=\frac{1}{M \times N} \sum_{x=1}^{M} \sum_{y=1}^{N}\left|I_{i j}(x, y)-\mu_{i j}\right|^{2}
\end{array}
$$

The feature vector of a face is then constructed by concatenating each block measure to one big feature vectors. This approach extracts the most discriminative features as well as reduces the feature vector size. The Euclidean distance metric is applied for classification.

In this paper in order to enhance the performance of Local DT-CWT the feature vector comparison is done on the basis of local parallelogram non overlapping windows of the subband face structure instead of rectangular or square windows. The arrangement of a parallelogram window is different from a rectangular window as seen in Figure 4.

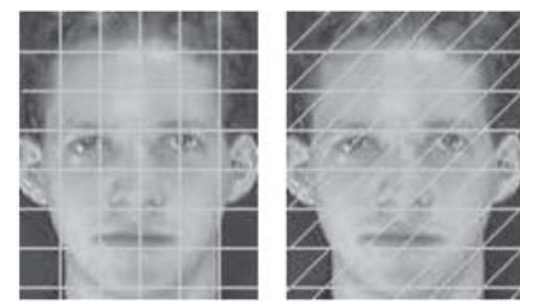

Fig. 4. Squared and Parallelogram Grid.

The perspective variation can be solved with the concept of parallelogram window based feature vectors. The combined feature vector from right and left direction parallelogram window provides better performance than the traditional square or rectangular window for the perspective variation.

\section{EXPERIMENTAL RESULTS}

This section evaluates the performance of Local DTCWT on Single Sample Problem. Two standard databases are used for evaluation of these results. The first database is ORL [14] database that contains a set of faces taken between April 1992 and April 1994 at the Olivetti Research Laboratory in Cambridge, UK. One of the persons is shown in Figure 5.

There are 10 different images of 40 distinct subjects. For some of the subjects, the images were taken at different times, varying lighting slightly, facial expressions (open/closed eyes, smiling/non-smiling) and facial details (glasses/no-glasses).

All the images are taken against a dark homogeneous background and the subjects are in up-right, frontal position (with tolerance for some side movement). The size of each image is $92 \times 112$, 8-bit grey levels. In this paper experiments were performed with one normal training image and 9 test images for each person.
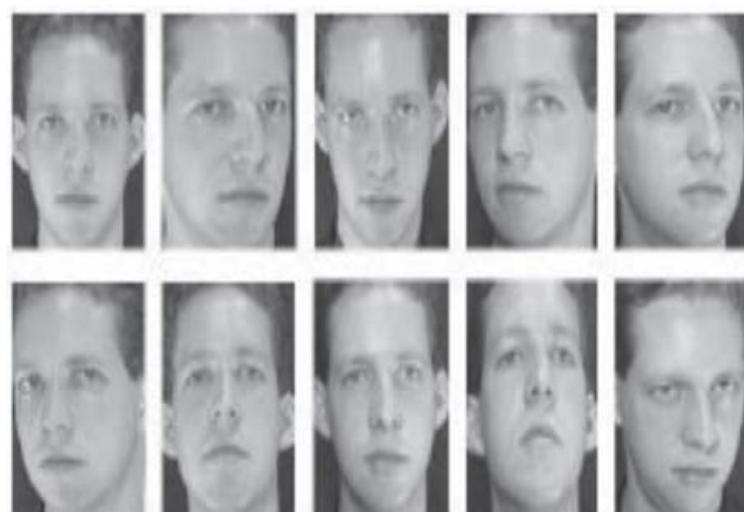

Fig. 5. One person in the ORL database 
The Yale face[15] database consists of 15 individuals, where for each individual, there are 11 face images containing variations in illumination and facial expression. From these 11 face images, we use frontal single face for training and the remaining 10 images are used for testing. Figure 6 depicts some sample images from the Yale database.

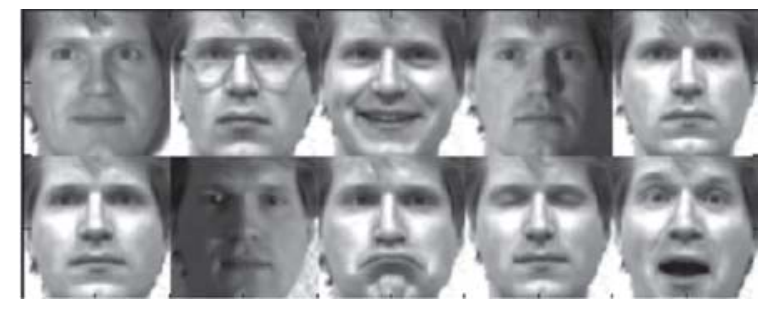

Fig. 6. Fig. 5. Faces from the YALE Face Database.

The experimental results presented in this section are divided into two parts. We make a comparison of Local DTCWT on square image structure as well as Local DT-CWT on parallelogram image structure with DT-CWT, PCA and $\mathrm{GW}+\mathrm{PCA}$. The respective performances of the above described methods based on the expression, illumination and perspective variant faces with single training image are shown in Table 1.

Table 1. Results on expression, ILLUMINATION AND perspective invariant face recognition with single sample per class

\begin{tabular}{|c|l|l|}
\hline \multirow{2}{*}{ Methods } & \multicolumn{2}{|c|}{ Experimental Datasets } \\
\cline { 2 - 3 } & \multicolumn{1}{|c|}{ ORL } & \multicolumn{1}{c|}{ Yale } \\
\hline PCA & $71 \%$ & $80 \%$ \\
\hline GW+PCA & $74.2 \%$ & $87.3 \%$ \\
\hline DT-CWT & $76.59 \%$ & $88.59 \%$ \\
\hline Local DT-CWT $(\mu, \sigma)$ & $78.44 \%$ & $90.33 \%$ \\
\hline $\begin{array}{c}\text { Local DT-CWT }(\mu, \sigma) \\
\text { Parallelogram Block }\end{array}$ & $81.78 \%$ & $92.62 \%$ \\
\hline
\end{tabular}

From the above result we can see that The Local DT-CWT on Square structure method outperforms DT-CWT with $1.85 \%$ for ORL dataset and $1.74 \%$ for Yale dataset. The Local DT-CWT on parallelogram structure method outperforms DT-CWT with $5.19 \%$ for ORL dataset and $4.03 \%$ for Yale Dataset. We can see from the Table 1, that our enhanced local DT-CWT based low level statistical feature based description has improved the DT-CWT performance greatly in this experiment.

Computational complexity: The face recognition program is performed with a Intel Pentium(R) D 2.40GHz CPU and
512GB RAM with Matlab7.0. Table 2 shows preliminary results of the average time for feature extraction of one face image and matching between an image pair.

Table 2: Comparison of Computational Complexity

\begin{tabular}{|c|l|l|}
\hline Average Time(ms) & Gabor & Local DT-CWT \\
\hline Feature extraction & 32 & 4 \\
\hline Matching & 0.44 & 0.03 \\
\hline
\end{tabular}

\section{CONCLUSIONS}

In this paper, we have proposed a novel approach of Local dual tree complex wavelet based facial feature description for human face recognition. Yale and ORL datasets are used, which contains the face images with different orientations, expressions and illumination. In our approach, the input face image is decomposed using dual tree complex wavelet transform. The recognition is done on the basis of euclidean distance measure on parallelogram image structure. Compared to the traditional Gabor-based methods, the LDT-CWT based method not only performs better, but also inherits low computational complexity.

This paper has also evaluated the performances of the LDT-CWT and the LDT-CWT on parallelogram image structure methods in terms of normal and changes in illumination, perspective and facial expressions faces of Yale face dataset and ORL datasets. Compared to LDTCWT defined on square structure, LDT-CWT on parallelogram structure has more potential for perspective invariant face recognition. We observe the results of face recognition using this approach are very good and encouraging. Furthermore, only one image per person is used for training which makes it useful for practical face recognition applications.

\section{ACKNOWLEDGMENT}

The comments and suggestions from the anonymous reviewers greatly improve this paper.

\section{REFERENCES}

[1] W.Zhao, R. Chellappa, A. Rosenfeld, and P.J.Phillips, "Face Recognition: A Literature Survey", Technical Report CAR-TR-948, Univ. of Maryland,CfAR,(2000).

[2] M. Turk and A. Pentland, "Eigenfaces for recognition," J. Cogn. Neurosci., vol. 13, no. 1, pp. 71-86, (1991).

[3] P. Nicholl and A. Amira, "DWT/PCA face recognition 
using automatic coefficient selection," in Proceedings of the 4th IEEE International Workshop on Electronic Design, Test and Applications (DELTA '08), pp. 390-393, Hong Kong, January 2008.

[4] L. Shen, Z. Ji, L. Bai, and C. Xu, "DWT based HMM for face recognition," Journal of Electronics, vol. 24, no. 6, pp. 835-837, 2007.

[5] A. Manjunath, R. Chellappa, and C. von der Malsburg, "A feature based approach to face recognition," in Proceedings of the IEEE Computer Society Conference on Computer Vision and Pattern Recognition (CVPR '92), pp. 373-378, Champaign, III, USA, June 1992.

[6] L. Shen, L. Bai, andM. Fairhurst, " Gabor wavelets and general discriminant analysis for face identification and verification," Image and Vision Computing, vol. 25, no. 5, pp. 553-563, 2007.

[7] Wenchao Zhang, Shiguang Shan, WenGao, Xilin Chen, Hongming Zhang, Jianyu Wang Local Gabor Binary Pattern Histogram Sequence (LGBPHS): a Novel Non-Statistical Model for Face Representation and Recognition Proceedings of the 10th International Conference on Computer Vision, pp. 150-155, October 15-21, (2005) , Beijing ,China
[8] Al-Amin Bhuiyan, and Chang Hong Liu, On Face Recognition using Gabor Filters proceedings of world academy of science, engineering and technology volume 22 july 2007 issn 1307-6884.

[9] Chengjun Liu, and Harryechsler. (Jul 2003) Independent Component Analysis of Gabor Features for Face Recognition IEEE Transactions On Neural Networks, vol. 14, no. 4, pp.919-928.

[10] H.K. Ekenel, B. Sankur, Multiresolution Face Recognition, Image and Vision Computing, vol. 23, 5, s.l., Elsevier Verlang, 2005, pp. 469-477.

[11] I. W. Selesnick, R. G. Baraniuk, and N. G. Kingsbury. The dual-tree complex wavelet transform. IEEE Signal Process. Mag., 05(22):123-151, November 2005.

[12] J. Yang, Y.Wang,W. Xu, and Q. Dai. Image coding using dual-tree discrete wavelet transform. IEEE Trans. Image Process., accepted, 2008.

[13] T. Ceil, H. Ozkaramanli, and H. Demirel. Facial feature extraction using complex dual-tree wavelet transform. Comput. Vis. Image Understand. (2008),doi10.1016/j.cviu.2007.12.001.

[14] TheORLdatabasehttp://www.cl.cam.ac.uk/ research/dtg/attarchive/facedatabase.html.

[15] Yale:http://cvc.yale.edu/projects/yalefaces/ yalefaces.html. 